\title{
Optical Nanofilters Based on Meta-Atom Side-Coupled Plasmonics Metal- Insulator-Metal Waveguides
}

\author{
Fu Sheng Ma and Chengkuo Lee, Member, IEEE
}

\begin{abstract}
We proposed a nanoplasmonic optical filtering technique based on complementary split-ring resonator structures. Interestingly, the proper plasmonic modes of the nanoring in the side-coupled arrangement can be selected and excited by the proposed structures. It is observed that the non-integer modes can be excited due to the presence of a metallic nano-wall as well as the integer modes. Furthermore, the numerical results indicate that the optical transmission spectrum of the investigated filter can be efficiently modified and tuned by manipulation either the position or the width of the employed nano-wall inside the metal-insulator-metal ring. The antinodes of the magnetic field of these modes, located on the symmetry plane of the proposed structures, can be manipulated by the position of the wall. Additionally, these modes, in particular the fundamental mode, are highly sensitive to the nano-wall dimensions. It indicates that the proposed nanofilter is a promising candidate as a tunable filter in nanophotonics applications.
\end{abstract}

Index Terms-Filters, plasmonics, metamaterials, waveguides.

\section{INTRODUCTION}

$\mathbf{N}$ OWADAYS, the bandwidth-limited electrical interconnects can no longer meet the increasing need for huge bandwidth and throughput capabilities in data center and high performance computing environments. To overtake the bottleneck in traffic exchange imposed by electrical wires, a clearly shaped roadmap is to bring optics into the spotlight and replacing electrical feedthrough with optical interconnects [1]. Since the limitation of the fundamental law of diffraction, the dimensions of photonic devices inevitably prevent the way towards high density integration for interfacing with electronics at the nanoscale. Plasmonics is a promising disruptive technology to fill the gap in size between photonic and electronic components. It also promises additional reductions in circuit size and increase in energy efficiency [2]-[4]. Plasmonics rely on the excitation of surface plasmon polaritons (SPPs) that are electromagnetic waves coupled to oscillations of free electrons in a metal and propagate along a metal-dielectric interface at near

Manuscript received January 22, 2013; revised May 27, 2013; accepted July 29, 2013. Date of publication August 02, 2013; date of current version August 12,2013 . This work was supported by MOE2012-T2-2-154 (Monolithic Integrated Si/AIN Nanophotonics Platform for Optical NEMS and OEICs) under WBS No. R-263-000-A59-112.

The authors are with the Department of Electrical and Computer Engineering, National University of Singapore, Singapore 117576 (e-mail: Fusheng.Ma@gmail.com; elelc@nus.edu.sg).

Color versions of one or more of the figures in this paper are available online at http://ieeexplore.ieee.org.

Digital Object Identifier 10.1109/JLT.2013.2275950 the speed of light [2]-[4]. The electromagnetic field intensity of SPPs reaches its maximum value at the metal surface and decays exponentially while moving away from the metal-dielectric interface [5]. Therefore, this strong intrinsic confinement is feasible even at sub-wavelength scale [6], breaking the size barriers of diffraction-limited photonics and enabling the development of compact integrated nanophotonic circuits [7].

Plasmonic waveguides based on the principles of SPPs have motivated significant activities to explore their characteristics for high integration benefitting from their ability of confining and guiding optical field in sub-wavelength scale. Among various types of plasmonic waveguides, an easily amenable structure (both analytically and experimentally) offering good confinement and acceptable propagation length are metal-insulatormetal (MIM) waveguides [8]-[12], where the mode is confined to the dielectric core in the form of a coupled gap-SPP between the two interfaces. Due to its unique characteristics including the strong confinement of optical field in nano-scale gaps, the high sensitivity of its transmission characteristics to the waveguide structures, and the facility of its fabrication, the MIM waveguide has attracted a great deal of effort in the fields of waveguide couplers [13], [14], sub-wavelength scale light confinement [15], [16], wavelength filters [17], [18], and integrated optical devices [19], [20]. Recently, numerous studies have been taken out to investigate the plasmonic resonators such as the plasmonic stubs [21]-[23], nano-capillary resonators [24], side-coupled FabryPerot [25], [26], high-performance wavelength demultiplexer [27], ultrafast all-optical switching [28], tunable high-channelcount bandpass filters [29], and slot cavities [30]-[34]. Ring resonator structure with circular or rectangular geometries is the mostly commonly used type of plasmonic resonators [35]-[40]. Recently, a nanoplasmonic optical filter based on a complementary square split-ring resonator (CSSRR) structure is proposed [41]. By including a metallic nano-wall, the non-integer modes can be excited besides the integer modes.

In this work, we investigate the optical filter structures in the form of the complementary circular split-ring resonator (CCSRR) side-coupled with a MIM bus waveguide. The CCSRR structure is formed by positioning a metallic nano-wall inside a MIM circular-ring, resulting in a widely studied complement structure known as split-ring resonators [42]-[46]. The resonance modes can be remarkably suppressed or excited by proper positioning of the wall. The influences of the nano-wall on the filtering characteristics are numerically investigated by the two dimensional finite-difference time-domain (FDTD) method with the perfectly matched layer (PML) boundary 


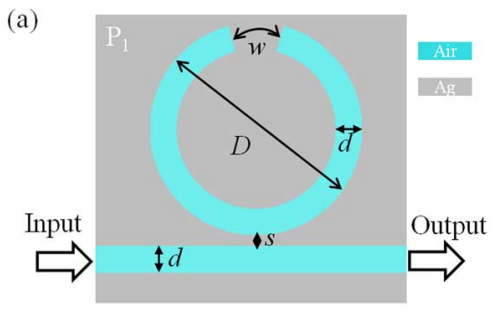

(b)

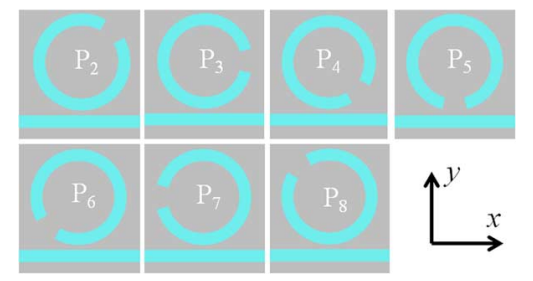

Fig. 1. Schematic of the proposed 2D plasmonic filter based on the CCSRR structure including a metallic nano-wall placed in the position of (a) $\mathrm{P}_{1}$, and (b) $\mathrm{P}_{2}-\mathrm{P}_{8}$

conditions [47]. From the simulation results, we found that not only the specific resonance modes of the circular MIM nanoring, but also the interesting non-integer resonant modes can be excited by proper positioning of a metallic nano-wall. However, it is not possible to excite these non-integer modes in the regular ring. They are found to be highly sensitive to the nano-wall dimensions and simply can be manipulated by varying the width of the nano-wall. For the CSSRR structure [41], in which the corners of the square ring can perturb the resonance modes and this perturbation effect need to be considered in the analysis. In contrast, for the CCSRR structure proposed here, it is not necessary to consider the perturbation effect of the corners existence without losing the excitation of the non-integer modes. The newly excited non-integer modes, which are not achievable by the regular ring, are found to be highly sensitive to the nano-wall dimensions. In contrast, the regular integer modes, in particular the high-order modes show less sensitivity to the variations of the nano-wall parameters. Hence, these non-integer modes of high sensitivity could be potentially used in designing high sensitive sensors, splitters, switches, and filters [3], [9], [11], [17]. Furthermore, the excitation of non-integer modes in the proposed CCSRR structures may also provide an alternative way for designing tunable multi-channel filtering devices based on an analogue of electromagnetically induced transparency [29].

\section{Modeling AND Simulation Method}

Fig. 1 shows the schematic drawings of the proposed structure. The circular air ring is surrounded by the silver (Ag) region. This ring is excited by a MIM plasmonic bus-waveguide in the side-coupled arrangement as shown in Fig. 1(a) and (b). The MIM circular ring is split by positioning a metallic nano-wall making the structure a complement to the metallic SRR. In this work, the nano-wall is placed at eight different positions, denoted by $\mathrm{P}_{1}, \mathrm{P}_{2}, \mathrm{P}_{3}, \mathrm{P}_{4}, \mathrm{P}_{5}, \mathrm{P}_{6}, \mathrm{P}_{7}$, and $\mathrm{P}_{8}$ [Fig. 1(a) and (b)]. The main structural parameters of the filter are the width of the MIM waveguide $(d)$, gap size between the bus-waveguide and the ring $(s)$, out diameter of the ring $(D)$, and width of the nano-wall $(w)$. Here the parameters $d, s, D$ are set to be $50 \mathrm{~nm}$, $20 \mathrm{~nm}$, and $400 \mathrm{~nm}$, respectively. Only a single propagation mode $\mathrm{TM}_{0}$ exists in the structure since widths of the waveguides are generally much less than the incident wavelength. The propagation constant $\beta$ of the $\mathrm{TM}_{0}$ can be obtained by solving the following dispersion equation [12]:

$$
\varepsilon_{d} k_{m}+\varepsilon_{m} k_{d} \tanh \left(\frac{k_{d} \omega}{2}\right)=0 .
$$

where $k_{m}=\left(\beta^{2}-\varepsilon_{m} k_{0}^{2}\right)^{1 / 2}, k_{d}=\left(\beta^{2}-\varepsilon_{d} k_{0}^{2}\right)^{1 / 2}, \varepsilon_{d}$ and $\varepsilon_{m}$ are the dielectric constants of the insulator and the metal, respectively. $k_{0}$ is the free space vector which equals to $2 \pi / \lambda$. The width of the MIM waveguides $(d)$ is set to be $50 \mathrm{~nm}$ to ensure that only the fundamental TM mode is supported. The insulator is assumed to be air $\left(\varepsilon_{i}=1\right)$, and the complex relative permittivity of silver $\varepsilon_{m}(\omega)$ is characterized by the well-known Drude model:

$$
\varepsilon_{m}(\omega)=\varepsilon_{\infty}-\frac{\omega_{p}^{2}}{\omega^{2}+i \gamma \omega} .
$$

where $\varepsilon_{\infty}$ is the dielectric constant at the infinite frequency, and $\omega$ is the angular frequency of incident light in vacuum. In the case of silver, we have $\varepsilon_{\infty}=3.7, \omega_{p}=9.1 \mathrm{eV}$, and $\gamma=0.018$ $\mathrm{eV}$ [48].

The 2D FDTD simulations are performed by using Lumerical FDTD Solution software with perfectly matched layer (PML) absorbing boundary conditions around all sides. In the following simulation, we specify the desired PML reflection as 0.0001 , rather than specifying the PML thickness. The grid sizes in $x$ and $y$-directions are chosen to be $\Delta x=\Delta y=4 \mathrm{~nm}$ to ensure a good convergence of the numerical calculations. The temporal step is $\Delta t=\Delta x / 2 c$, where $c$ is the velocity of light in vacuum. Since the width of the bus waveguide is much smaller than the incident wavelength, only the fundamental TM mode is excited in the structure. The fundamental TM mode of the MIM plasmonic waveguide is generated at the left end of the bus-waveguide and injected along the $x$-axis, and the output monitor is located at the right end of the bus-waveguide. When a plane wave with TM polarization is incident into the proposed structure, the transmitted power flows $P=\left[\int \operatorname{real}(E \times H *) \mathrm{d} S\right] / 2$, which are integrated over the cross section of the waveguides, are recorded as a function of time at the output monitor. Finally, the recorded transmission powers are post-processed with Fourier transform in order to obtain the transmission spectra of the structure.

\section{Simulation Results And Discussions}

To investigate the effect from the metal nano-wall placed in the MIM ring, we firstly calculated the transmission spectrum of the regular circular ring (Fig. 2(a)) and spectrum of the proposed CCSRR filter with the nano-wall $(w=20 \mathrm{~nm})$ placed at position $\mathrm{P}_{1}$ (Fig. 2(b)). The magnetic field distributions of the resonance modes are shown as insets in Fig. 2. Here, the resonance modes of the regular circular ring are denoted as $\mathrm{TM}_{m}$, and accordingly the resonance modes of the CCSRR filters are denoted as $\mathrm{TM}_{m}\left(\mathrm{P}_{n}\right)$, where " $m$ " and " $\mathrm{P}_{n}$ " stand for the type of the mode and the position of the nano-wall, respectively. As shown in Fig. 2(b), there are extra resonances in the transmission spectrum of the CCSRR filter with the nano-wall placed 


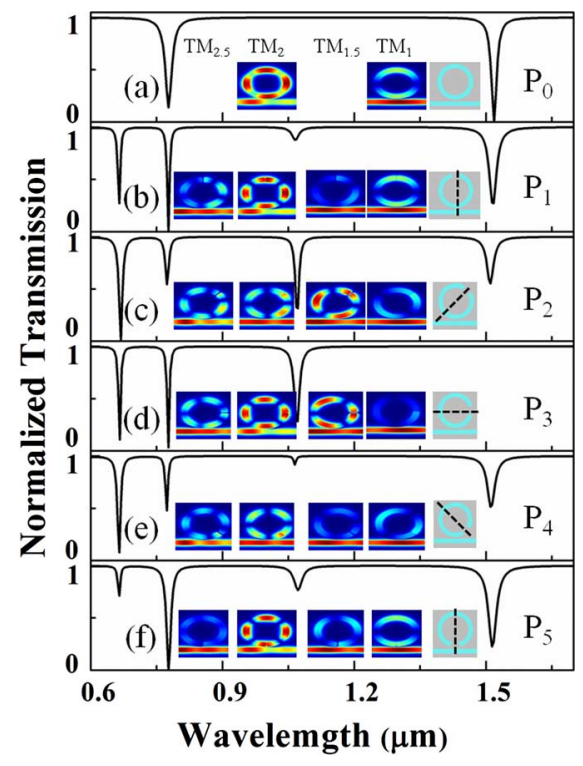

Fig. 2. Transmission spectrum of the filter comprises a regular circular ring and the spectra of the proposed CCSRR filter with a nano-wall placed in various positions $\mathrm{P}_{1}-\mathrm{P}_{5}$, calculated using the FDTD method. The insets are the magnetic-field distributions at the resonance wavelengths. The dash lines indicate the symmetric plane of the CCSRR filter.

at position $\mathrm{P}_{1}$ as well as the regular modes of the circular ring. Actually, the proposed plasmonic CCSRR can be considered as a closed straight MIM cavity [26] which is bent, and its ends are separated by the wall. Based on this analogy the CCSRR can support both the integer modes (including even number of antinodes) and the non-integer modes (including odd number of antinodes). It can be found from the magnetic-field distributions of the resonance modes that the circular ring only supports the integer modes $\mathrm{TM}_{1}$ and $\mathrm{TM}_{2}$. While for the CCSRR, not only the integer modes $\mathrm{TM}_{1}\left(\mathrm{P}_{1}\right)$ and $\mathrm{TM}_{2}\left(\mathrm{P}_{1}\right)$ but also the non-integer modes $\mathrm{TM}_{1.5}\left(\mathrm{P}_{1}\right)$ and $\mathrm{TM}_{2.5}\left(\mathrm{P}_{1}\right)$ are observed. The antinodes of the magnetic field of these resonance modes are located on the symmetry plane of the regular ring and the proposed CCSRR. By incorporating the metallic nano-wall, the magnetic fields on the sides of the wall can be arranged either with the same (integer modes) or opposite (non-integer modes) polarities. By comparing the resonance wavelengths and the field profiles of the integer and non-integer modes as shown in Fig. 2, it is observed that the integer modes $\mathrm{TM}_{1}\left(\mathrm{P}_{1}\right)$ and $\mathrm{TM}_{2}\left(\mathrm{P}_{1}\right)$ are almost the same as their counterpart modes in the regular circular ring.

The transmission spectra and the mode profiles of the resonance modes with the nano-wall placed at positions $\mathrm{P}_{2}, \mathrm{P}_{3}, \mathrm{P}_{4}$ and $\mathrm{P}_{5}$ are shown in Fig. 2(c)-(f). The spectrum clearly depends on the position of the wall. The resonance wavelengths of the excited integer and non-integer modes are almost independent on the positions of the nano-walls. However, the intensity of the resonances is remarkably affected. For instance, the maximum intensity of $\mathrm{TM}_{1.5}$ modes is observed when the nano-wall is placed at position $\mathrm{P}_{3}$. By comparing the mode profiles of the resonance modes, the insets as shown in Fig. 2, it is observed that the antinodes of the field patterns can be manipulated by the position of the wall. The antinodes of the field profile of the resonance modes locate on the symmetry plane of the CCSRR.

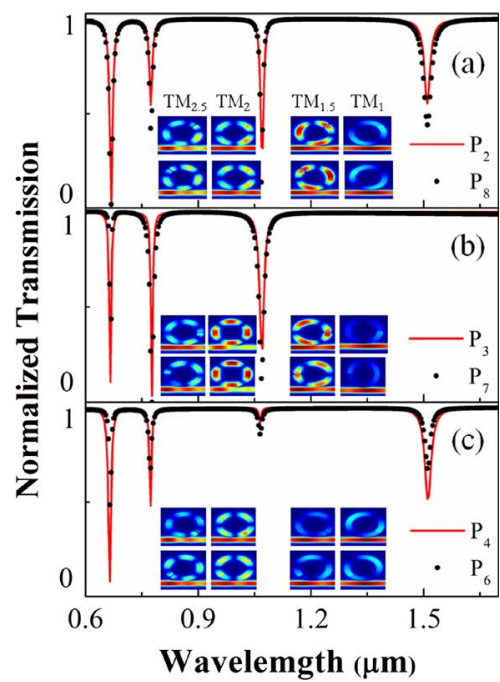

Fig. 3. Transmission spectra of the proposed CCSRR filters with a nano-wall placed at positions (a) $\mathrm{P}_{2}$ and $\mathrm{P}_{8}$, (b) $\mathrm{P}_{3}$ and $\mathrm{P}_{7}$, and (c) $\mathrm{P}_{4}$ and $\mathrm{P}_{6}$, calculated using the FDTD method. The insets are the magnetic-field distributions at the resonance wavelengths.

This interesting feature of incorporating the metallic nano-wall can be understood by the fact that the efficient extraction of optical power from a resonator occurs when the extracting waveguide is at the position of antinodes of the magnetic fields for the MIM configuration.

According to the above discussion, we can manipulate the excited classes and thereby modify the transmission spectrum of the filter by placing a metallic nano-wall on the symmetry planes of the circular ring. To investigate the influence of the positions of the nano-wall on the response of the filter, eight proper positions for the wall are considered as shown in Fig. 1(a) and (b). However, due to the symmetrical plane of the proposed structures along the $y$ axis, the transmission spectra of CCSRR with the nano-walls symmetrically placed about the $y$ axis are similar, for instance, the spectra with wall placed at $\mathrm{P}_{2}$ and $\mathrm{P}_{8}$ as shown in Fig. 3(a). Although the resonance wavelengths of the modes are similar, the magnetic field distribution is different with the antinodes of the field profile located on the symmetry plane of the CCSRR. A similar phenomenon is also observed between $\mathrm{P}_{3}$ and $\mathrm{P}_{7}$, and between $\mathrm{P}_{4}$ and $\mathrm{P}_{6}$ as shown in Figs. (b) and (c), respectively.

As discussed above, the transmission spectra of the proposed CCSRR filters are drastically affected by the positions of the nano-wall. The width of the nano-wall is also expected to remarkably influence the resonance wavelengths of the filters. The dependence of the resonance wavelengths of the integer and non-integer modes on the width of the wall, $w$ at position $\mathrm{P}_{1}-\mathrm{P}_{5}$ is shown in Fig. 4(a)-(e). Along with the width of the nano-wall $w$ varies from 20 to $245 \mathrm{~nm}$, it can be observed that the variation of the resonance wavelength of the $\mathrm{TM}_{1}\left(\mathrm{P}_{1}\right), \mathrm{TM}_{1.5}\left(\mathrm{P}_{1}\right)$, $\mathrm{TM}_{2}\left(\mathrm{P}_{1}\right)$, and $\mathrm{TM}_{2.5}\left(\mathrm{P}_{1}\right)$ modes are $238 \mathrm{~nm}, 210 \mathrm{~nm}, 110 \mathrm{~nm}$ and $114 \mathrm{~nm}$, respectively. The variations in the resonance wavelengths of the $\mathrm{TM}_{1}\left(\mathrm{P}_{1}-\mathrm{P}_{5}\right), \mathrm{TM}_{1.5}\left(\mathrm{P}_{1}-\mathrm{P}_{5}\right), \mathrm{TM}_{2}\left(\mathrm{P}_{1}-\mathrm{P}_{5}\right)$, and $\mathrm{TM}_{2.5}\left(\mathrm{P}_{1}-\mathrm{P}_{5}\right)$ modes with the width $w$ varies from 20 to $245 \mathrm{~nm}$ are summarized in Table I. Generally, the resonance wavelengths of the considered modes decrease by increasing the width $w$. Since the equivalent inductance for the wider wall is 
(a)

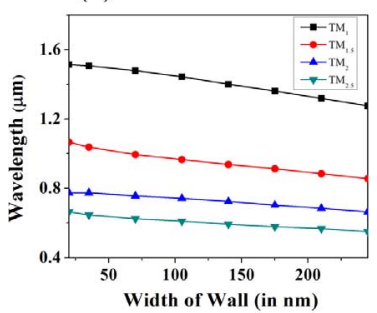

(c)

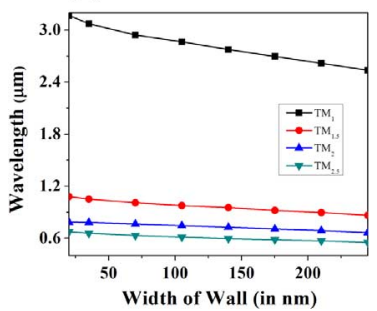

(e)

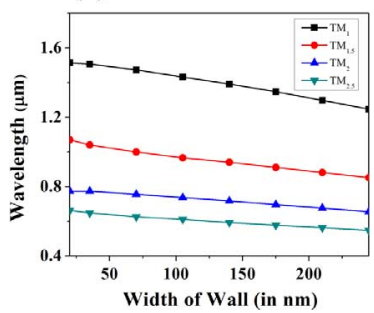

(b)

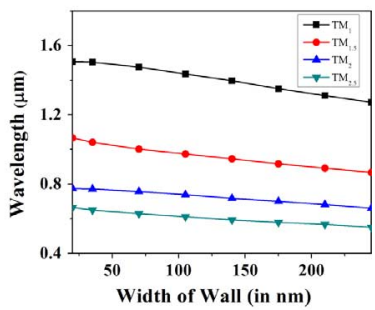

(d)

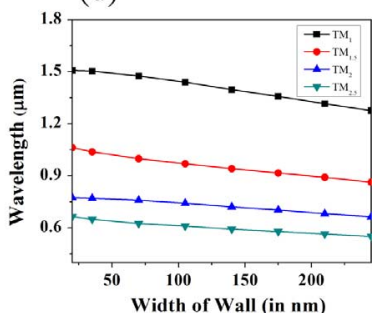

the filtering tunability of the proposed structures. The non-integer modes, which are not achievable for regular circular ring, are excited by having a metallic nano-wall within the MIM nano-ring structure. By manipulating the width of the nanowall, the fundamental mode is observed to be modified significantly. The resonance wavelength shift of about $630 \mathrm{~nm}$ is achieved for the fundamental mode of the CCSRR filters upon a gradual variation of the width of the wall from 20 to $245 \mathrm{~nm}$. Our findings show that our proposed CCSRR structures offers great flexibility for the design of tunable and ultra-compact optical components in integrated optical circuits and for nanophotonics applications.

\section{REFERENCES}

[1] D. Miller, "Device requirements for optical interconnects to silicon chips," Proc. IEEE, vol. 97, no. 7, pp. 1166-1185, Jul. 2009.

[2] W. L. Barnes, A. Dereux, and T. W. Ebbesen, "Surface plasmon subwavelength optics," Nature, vol. 424, pp. 824-830, 2003.

[3] H. A. Atwater, "The promise of plasmonics," Sci. Am., vol. 296, no. 4, pp. 56-63, Apr. 2007.

[4] M. L. Brongersma and V. M. Shalaev, "The case for plasmonics," Science, vol. 328, no. 5977, pp. 440-441, Apr. 2010.

[5] R. H. , Surface Plasmons on Smooth and Rough Surfaces and on Gratings. Berlin, Germany: Springer-Verlag, 1988.

[6] D. K. Gramotnev and S. I. Bozhevolnyi, "Plasmonics beyond the diffraction limit," Nat. Photon., vol. 4, no. 2, pp. 83-91, Jan. 2010.

[7] J. Grandidier, S. Massenot, A. Bouhelier, G. Colas des Francs, J.-C. Weeber, L. Markey, and A. Dereux, "Surface plasmon routing in dielectric-loaded surface plasmon polariton waveguides," Proc. SPIE, vol. 7033, pp. 70330S-8, Aug. 2008.

Fig. 4. Variation of the resonance wavelengths of the CCSRR versus the width of the metallic nano-wall placed in positions: (a) $\mathrm{P}_{1}$, (b) $\mathrm{P}_{2}$, (c) $\mathrm{P}_{3}$, (d) $\mathrm{P}_{4}$ and (e) $\mathrm{P}_{5}$, calculated by the FDTD method.

TABLE I

VARIATIONS IN THE RESONANCE WAVELENGTH OF THE TM $\mathrm{TM}_{1}\left(\mathrm{P}_{1}-\mathrm{P}_{5}\right)$, $\mathrm{TM}_{1.5}\left(\mathrm{P}_{1}-\mathrm{P}_{5}\right), \mathrm{TM}_{2}\left(\mathrm{P}_{1}-\mathrm{P}_{5}\right)$, AND $\mathrm{TM}_{2.5}\left(\mathrm{P}_{1}-\mathrm{P}_{5}\right)$ MODES

\begin{tabular}{ccccc}
\hline \hline \multirow{2}{*}{$\begin{array}{c}\text { Nano-wall } \\
\text { Position }\end{array}$} & \multicolumn{3}{c}{ Variation of resonance wavelength (in $\mathrm{nm}$ ) } \\
\cline { 2 - 5 } & $\mathrm{TM}_{1}$ & $\mathrm{TM}_{1.5}$ & $\mathrm{TM}_{2}$ & $\mathrm{TM}_{2.5}$ \\
\hline $\mathrm{P}_{1}$ & 238 & 210 & 110 & 114 \\
$\mathrm{P}_{2}$ & 235 & 199 & 114 & 114 \\
$\mathrm{P}_{3}$ & 633 & 217 & 121 & 125 \\
$\mathrm{P}_{4}$ & 232 & 199 & 111 & 114 \\
$\mathrm{P}_{5}$ & 267 & 215 & 119 & 116 \\
\hline \hline
\end{tabular}

smaller, a large inductive behavior of a narrow metallic wall may result in a large change in the resonant wavelengths. Additionally, the results show that the sensitivity of the resonance wavelengths of both the integer and the non-integer modes to the variation of the wall width decreases when the mode number increases. By comparing with the variation of the integer and the non-integer modes, the resonant modes of the CCSRR with nano-wall at position $\mathrm{P}_{3}$ are highly sensitive to the variation of the nano-wall width.

\section{CONCLUSION}

In summary, a 2-D plasmonic nanofilter based on the CCSRR structure is proposed and validated computationally. The numerical simulation results demonstrate the mode-selectivity and
[8] G. Veronis and S. Fan, "Crosstalk between three-dimensional plasmonic slot waveguides," Opt. Exp., vol. 16, no. 3, pp. 2129-40, Feb. 2008.

[9] W. Cai, W. Shin, S. Fan, and M. L. Brongersma, "Elements for plasmonic nanocircuits with three-dimensional slot waveguides," Adv.

[10] D. F. P. Pile, T. Ogawa, D. K. Gramotnev, Y. Matsuzaki, K. C. Vernon, K. Yamaguchi, T. Okamoto, M. Haraguchi, and M. Fukui, "Two-dimensionally localized modes of a nanoscale gap plasmon waveguide," Appl. Phys. Lett., vol. 87, no. 26, p. 261114, 2005.

[11] G. Veronis and S. Fan, "Bends and splitters in metal-dielectric-metal subwavelength plasmonic waveguides," Appl. Phys. Lett., vol. 87, no. 13, p. 131102, 2005.

[12] J. Dionne, L. Sweatlock, H. Atwater, and A. Polman, "Plasmon slot waveguides: Towards chip-scale propagation with subwavelength-scale localization," Phys. Rev. B, vol. 73, no. 3, pp. 1-9, Jan. 2006.

[13] T.-W. Lee and S. Gray, "Subwavelength light bending by metal slit structures," Opt. Exp., vol. 13, no. 24, pp. 9652-9659, Nov. 2005.

[14] R. A. Wahsheh, Z. Lu, and M. A. G. Abushagur, "Nanoplasmonic couplers and splitters," Opt. Exp., vol. 17, no. 21, pp. 19033-19040, Oct. 2009.

[15] K. Y. Kim, Y. K. Cho, H.-S. Tae, and J.-H. Lee, "Light transmission along dispersive plasmonic gap and its subwavelength guidance characteristics," Opt. Exp., vol. 14, no. 1, pp. 320-330, Jan. 2006.

[16] Y. Jun, R. Kekatpure, J. White, and M. Brongersma, "Nonresonant enhancement of spontaneous emission in metal-dielectric-metal plasmon waveguide structures," Phys. Rev. B, vol. 78, no. 15, pp. 1-4, Oct. 2008.

[17] C. Min and G. Veronis, "Absorption switches in metal-dielectric-metal plasmonic waveguides," Opt. Exp., vol. 17, no. 13, pp. 10757-10766, Jun. 2009.

[18] S. I. Bozhevolnyi, V. S. Volkov, E. Devaux, J.-Y. Laluet, and T. W. Ebbesen, "Channel plasmon subwavelength waveguide components including interferometers and ring resonators," Nature, vol. 440, no. 7083, pp. 508-511, Mar. 2006.

[19] A. Pannipitiya, I. D. Rukhlenko, M. Premaratne, H. T. Hattori, and G. P. Agrawal, "Improved transmission model for metal-dielectric-metal plasmonic waveguides with stub structure," Opt. Exp., vol. 18, no. 6, pp. 229-232, 2010.

[20] M. L. Brongersma and S. Fan, "Metal-dielectric-metal plasmonic waveguide devices for manipulating light at the nanoscale Invited Paper," Chin. Opt. Lett., vol. 7, no. 4, pp. 302-308, 2009. Mater., vol. 22, no. 45, pp. 5120-5124, Dec. 2010. 
[21] Y. Matsuzaki, T. Okamoto, M. Haraguchi, M. Fukui, and M. Nakagaki, "Characteristics of gap plasmon waveguide with stub structures," Opt. Exp., vol. 16, no. 21, pp. 16314-16325, Oct. 2008.

[22] X.-S. Lin and X.-G. Huang, "Tooth-shaped plasmonic waveguide filters with nanometeric sizes," Opt. Lett., vol. 33, no. 23, pp. 2874-2876, Dec. 2008.

[23] J. Tao, X. G. Huang, X. Lin, Q. Zhang, and X. Jin, "A narrow-band subwavelength plasmonic waveguide filter with asymmetrical multipleteeth-shaped structure," Opt. Exp., vol. 17, no. 16, pp. 13989-13994, Aug. 2009.

[24] J. Tao, X. G. Huang, and J. H. Zhu, "A wavelength demultiplexing structure based on resonators," Opt. Exp., vol. 18, no. 11, pp. $11111-11116,2010$

[25] A. Noual, A. Akjouj, Y. Pennec, J.-N. Gillet, and B. Djafari-Rouhani, "Modeling of two-dimensional nanoscale Y-bent plasmonic waveguides with cavities for demultiplexing of the telecommunication wavelengths," New J. Phys., vol. 11, no. 10, p. 103020, Oct. 2009.

[26] X. Mei, X. Huang, J. Tao, J. Zhu, Y. Zhu, and X. Jin, “A wavelength demultiplexing structure based on plasmonic MDM side-coupled cavities," J. Opt. Soc. Amer. B, vol. 27, no. 12, p. 2707, Nov. 2010.

[27] H. Lu, X. Liu, Y. Gong, D. Mao, and L. Wang, "Enhancement of transmission efficiency of nanoplasmonic wavelength demultiplexer based on channel drop filters and reflection nanocavities," Opt. Exp., vol. 19, no. 14, pp. 12885-12890, Jul. 2011.

[28] H. Lu, X. Liu, L. Wang, Y. Gong, and D. Mao, "Ultrafast all-optical switching in nanoplasmonic waveguide with Kerr nonlinear resonator,' Opt. Exp., vol. 19, no. 4, pp. 2910-2915, Feb. 2011.

[29] H. Lu, X. Liu, G. Wang, and D. Mao, "Tunable high-channel-count bandpass plasmonic filters based on an analogue of electromagnetically induced transparency," Nanotechnology, vol. 23, no. 44, p. 444003, 2012.

[30] G. Wang, H. Lu, X. Liu, D. Mao, and L. Duan, "Tunable multi-channel wavelength demultiplexer based on MIM plasmonic nanodisk resonators at telecommunication regime," Opt. Exp., vol. 19, no. 4, pp. 3513-3518, Feb. 2011

[31] H. Lu, X. Liu, D. Mao, L. Wang, and Y. Gong, "Tunable band-pass plasmonic waveguide filters with nanodisk resonators," Opt. Exp., vol. 18 , no. 17 , p. 17922 , Aug. 2010

[32] F. Hu, H. Yi, and Z. Zhou, "Wavelength demultiplexing structure based on arrayed plasmonic slot cavities," Opt. Lett., vol. 36, no. 8, pp. 1500-1502, Apr. 2011

[33] Y. Guo, L. Yan, W. Pan, B. Luo, K. Wen, Z. Guo, H. Li, and X. Luo, "A plasmonic splitter based on slot cavity," Opt. Exp., vol. 19, no. 15, pp. 13831-13838, Jul. 2011.

[34] K. Shang, L. Yan, K. Wen, Z. Guo, Y. Guo, W. E. I. Pan, and X. Luo, "Separation of resonance modes in nanoring resonator by a cascaded slot cavity," Mod. Phys. Lett. B, vol. 26, no. 23, pp. 1-8, 2012.

[35] S. Xiao, L. Liu, and M. Qiu, "Resonator channel drop filters in a plasmon-polaritons metal," Opt. Exp., vol. 14, no. 7, pp. 2932-2937, Apr. 2006.

[36] A. Hosseini and Y. Massoud, "Nanoscale surface plasmon based resonator using rectangular geometry," Appl. Phys. Lett., vol. 90, no. 18, p. 181102, 2007.
[37] T. Holmgaard, Z. Chen, S. I. Bozhevolnyi, A. Dereux, and N. B. All, "Dielectric-loaded plasmonic waveguide ring resonators," Opt. Exp., vol. 17, no. 4, pp. 2968-2975, 2009.

[38] Z. Han, V. Van, W. N. Herman, and P.-T. Ho, "Aperture-coupled MIM plasmonic ring resonators with sub-diffraction modal volumes," Opt. Exp., vol. 17, no. 15, pp. 12678-12684, July 2009.

[39] T.-B. Wang, X.-W. Wen, C.-P. Yin, and H.-Z. Wang, "The transmission characteristics of surface plasmon polaritons in ring resonator," Opt. Exp., vol. 17, no. 26, pp. 24096-24101, Dec. 2009.

[40] B. Yun, G. Hu, and Y. Cui, "Theoretical analysis of a nanoscale plasmonic filter based on a rectangular metal-insulator-metal waveguide," J. Phys. D: Appl. Phys., vol. 43, no. 38, p. 385102, Sept. 2010.

[41] I. Zand, A. Mahigir, T. Pakizeh, and M. S. Abrishamian, "Selectivemode optical nanofilters based on plasmonic complementary split-ring resonators," Opt. Exp., vol. 20, no. 7, pp. 7516-7525, 2012.

[42] T. D. Corrigan, P. W. Kolb, A. B. Sushkov, H. D. Drew, D. C. Schmadel, and R. J. Phaneuf, "Optical plasmonic resonances in split-ring resonator structures: An improved LC model," Opt. Exp., vol. 16, no. 24, pp. 19850-19864, Nov. 2008.

[43] C. Rockstuhl, T. Zentgraf, T. P. Meyrath, H. Giessen, and F. Lederer, "Resonances in complementary metamaterials and nanoapertures," Opt. Exp., vol. 16, no. 3, pp. 2080-2090, Feb. 2008.

[44] M. Navarro-Cía, M. Aznabet, M. Beruete, F. Falcone, O. El Mrabet, M. Sorolla, and M. Essaaidi, "Stacked complementary metasurfaces for ultraslow microwave metamaterials," Appl. Phys. Lett., vol. 96, no. 16, p. 164103, 2010.

[45] M. D. Design, Y. Dong, S. Member, T. Itoh, and L. Fellow, "Substrate integrated waveguide loaded by complementary split-ring resonators for," IEEE Microw. Wireless Compon. Lett., vol. 21, no. 1, pp. 10-12, 2011.

[46] G. Kumar, A. Cui, S. Pandey, and A. Nahata, "Planar terahertz waveguides based on complementary split ring resonators," Opt. Exp., vol. 19, no. 2, pp. 1072-1080, Jan. 2011.

[47] A. T. and S. C. Hagness, Computational Electrodynamics: The Finite-Difference Time-Domain Method. Norwood, MA, USA: Artech House, 2005.

[48] O. Constants, "Optical constants of the noble metals," Phys. Rev. B, vol. 6 , pp. 4370-4379, 1972.

Fu Sheng Ma (M’09) received the Ph.D. degree from the National University of Singapore, Singapore. He is currently a Research Fellow with the Department of Electrical and Computer Engineering, National University of Singapore, Singapore.

Chengkuo Lee (M'96) received the Ph.D. degree from The University of Tokyo, Bunkyo, Japan. He is currently an Associate Professor with the Department of Electrical and Computer Engineering, National University of Singapore, Singapore. 